\title{
Set lasers to image
}

\author{
Engineers and neuroscientists working to advance multiphoton microscopy are pushing the depths, speeds, and \\ scales of in vivo imaging.
}

\section{Ellen P. Neff}

ight is an exciting tool. If you shine light with a wavelength of about $488 \mathrm{~nm}$ at a cell engineered to express green fluorescent protein (GFP), that protein will get excited. Give it a few nanoseconds and the GFP will emit photons back, leaving the cell with a detectable green glow. This feature of GFP, and of other fluorophores and fluorescent indicators discovered and designed since, has enabled researchers to label structures of interest in a sample, such as neurons, and record their activity.

That includes in the neurons of living animals-particularly mice, where considerable investment in recent decades has been spent building the genetic tools to create animals that can express different fluorescent proteins, but also in other rodents such as rats, in an ever growing number of invertebrate species, and in larger nonhuman primates, including macaques and marmosets. "For imaging, that's been critical," says Jack Waters, an associate investigator at the Allen Institute in Seattle, WA.

But as researchers attempt to peer into the brains of their animals, they can find themselves losing the light. "The big challenge is the brain is opaque-when you look at it, you only see the surface," says Kaspar Podgorski, a fellow at the Howard Hughes Medical Institute Janelia Research Campus in Ashburn, VA. "Light in general is best at looking through transparent objects... Even something very, very thin quickly becomes opaque."

Traditional, single-photon confocal microscopes can resolve structures to a few 10 s of microns through a tissue. But when researchers want to look deeper, confocals can't cut it. "That's where multiphoton really comes in," says Podgorski. Multiphoton lasers can push the limits of in vivo imaging, with recent developments in 2 - and 3-photon microscopy allowing researchers to image deeper, faster, and over larger areas and volumes in vivo.

\section{To the depths}

The Multiphoton Imaging Core at Johns Hopkins University has a number of microscope set ups available for its neuroscience users. There are the breadand-butter confocal microscopes and

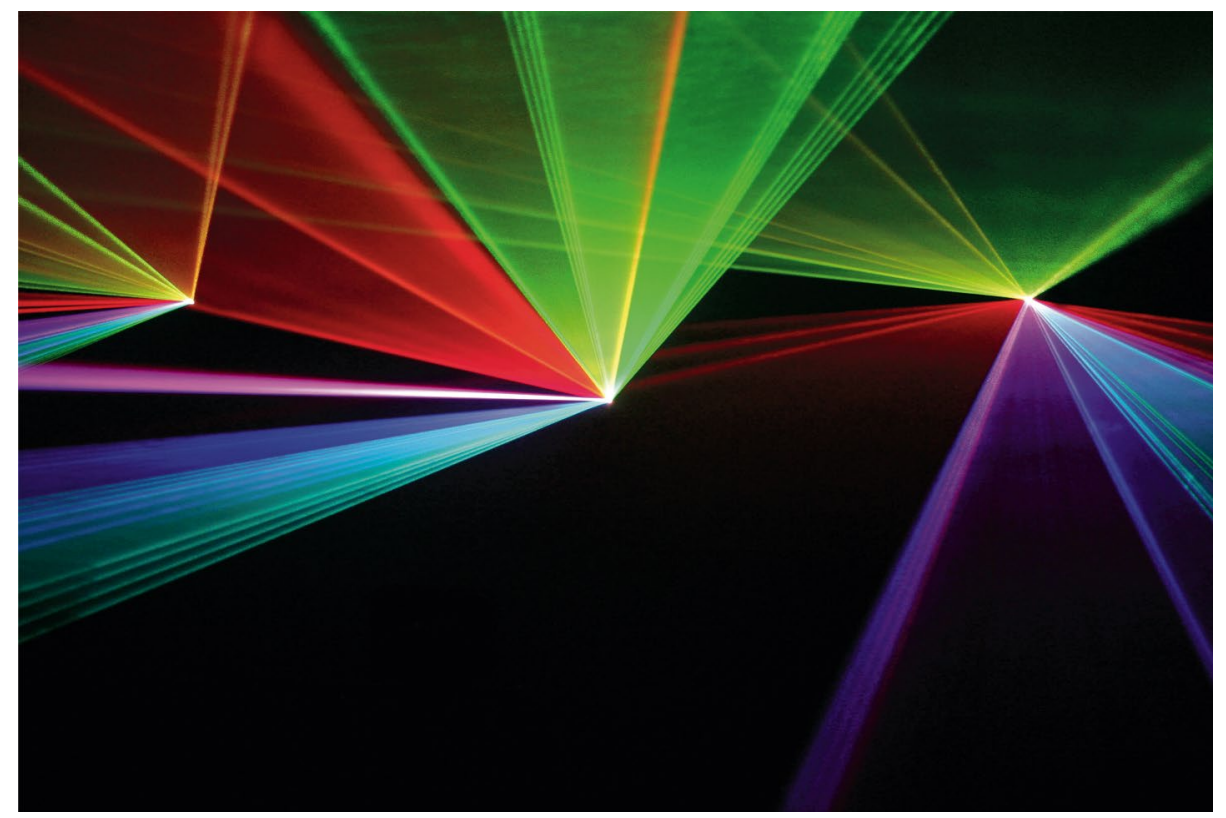

Image away! Lasers of different wavelengths of light can penetrate to different depths in vivo. Credit: ThomasIskra / Panther MediaGmBH / Alamy Stock Photo

several commercially available 2-photon (2P) instruments, as well as a recently added 3-photon (3P) system that the Core is currently trialing. Deciding which instruments a researcher should use comes down to depth, says Core director Michele Pucak. "For people who want to look at something that's right on the surface of the brain, they don't need 2 photon-they can just use regular old confocals," she says.

For shallow imaging, a seemingly simple microscope can be the best choice, as multiphoton lasers are inherently less efficient: two, or in case of 3P instruments, three photons of light have to hit the fluorescent molecule in just the right way and at just the right time to excite it. Confocal microscopes use visible wavelengths of light, and they work by exciting everything in their path; in thin samples, it's then easy enough to figure out where photons are emitted from to focus only on the structure of interest.

While you can focus a confocal microscope deep in a tissue, that tissue quickly gets in the way. "That light has to pass through all the surface structures," explains Waters, and it becomes near impossible to focus on a deep structure while ignoring fluorescence from those above it. Multiphoton microscopes solve this problem in a different way: they don't excite fluorescence in surface layers at all. $2 \mathrm{P}$ and 3P microscopy uses nonlinear excitation at the focus of the layer beam, which helps avoid that out-of-focus fluorescence, and longer wavelengths of light, which can penetrate deeper into tissues without being scattered. Think of shining a flashlight against your hand, says Podgorski: blue \& green wavelengths are absorbed or scattered, while the red passes through.

Whereas confocals emit visible wavelengths of light, $2 \mathrm{P}$ systems extend the wavelength to between 900 and 1000 $\mathrm{nm}$, which reduces scattering and makes the brain appear more transparent. The wavelength used can depend on the depth to be imaged and the fluorescent molecule used. GFP, for example, can be excited by 


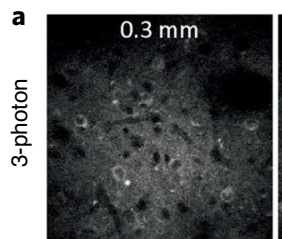

b

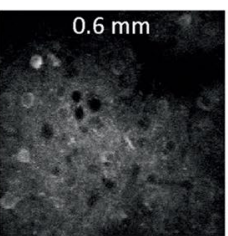

$300 \mu \mathrm{m}$
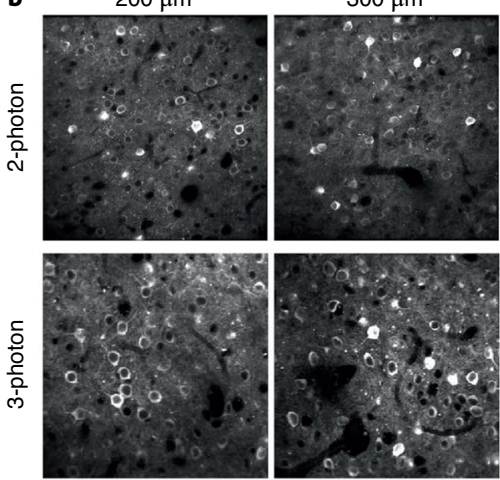

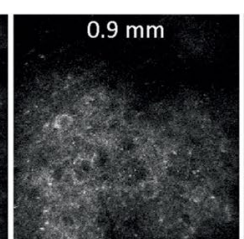

$400 \mu \mathrm{m}$
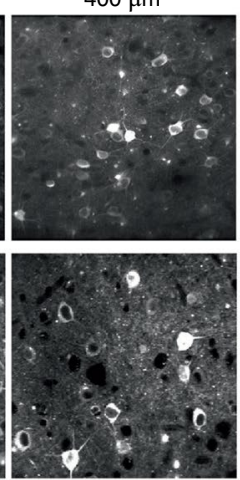

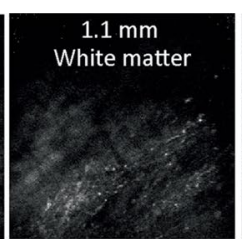

$500 \mu \mathrm{m}$
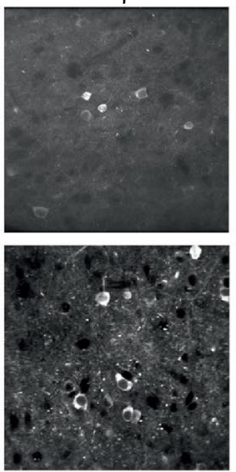

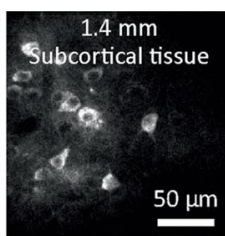

$600 \mu \mathrm{m}$

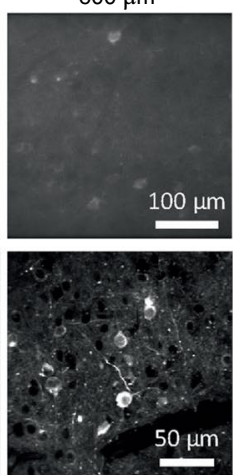

Finding the best fit | 3P imaging down to subcortical depths (a) and comparing 2P \& 3P microscopes at different focus depths (b) in the mouse cortex. Image reprinted with permission from Takasaki, T. (2020) ${ }^{1}$, Society for Neuroscience.

wavelengths of $488 \mathrm{~nm}$ or about $920 \mathrm{~nm}-$ that latter, deliverable by a $2 \mathrm{P}$ system, can make it to fluorescent molecules deeper in the tissue. Under ideal conditions, 2P microscopes can image up to about a millimeter into a sample-though in practice, depth can often be less than half that distance. For comparison, the cortex of a mouse spans between $0.8 \mathrm{~mm}$ and $1.2 \mathrm{~mm}$. "If you're interested in the cortex, then that's quite a lot," says Waters.

3P microscopy just ups the ante-3P lasers emit three photons of infrared light with wavelengths between $1300 \mathrm{~nm}$ and $1700 \mathrm{~nm}$, which can to penetrate upwards of a millimeter and half. In a mouse, that's the whole depth of its cortex and a little more, and with clearer contrast along the way ${ }^{1}$. "The image quality you get out of three photon microscopes is astounding, even to people who are accustomed to 2 photon images. 2 photon compared to confocal was like night and day, but there's a big step up again with 3 photon for many applications," says Waters.

But each has its own role, depending on the questions being asked: "There's a general principle, which is if you can, you do things with simpler system," says Waters. "If you need to go deeper than you can with 2 photon, then you're forced to use 3 photon microscopy. But if you can do your experiment with 2 photon microscopy, which is more mature, and a little cheaper to implement, then you're going to do your experiments with 2 photon microscopy."

\section{Wider, better, faster, stronger} A multiphoton instrument involves two main components: a laser and a microscope. "We build microscopes all the time, but making lasers is a whole new level of complexity," says Waters. Early 3P engineering labs, such as that of Chris Xu at Cornell, did build their own lasers, but commercial options have started becoming more available in the past couple of years.

"I started 2 photon microscopy in a world that feels very much like 3 photon microscopy does now-early stage lasers, which were a little bit difficult to handle," says Waters, though time, experience, and examples of its applications will likely bring more labs to that third photon. In the meantime, those in the multiphoton field are still pushing the capabilities of $2 \mathrm{P}$ microscopes, to image faster and at greater scales in vivo.

Podgorski's lab, for example, is interested in recording synaptic activity in mice in order to figure out the logic of how neurons transform inputs into outputs. A given neuron, however, will receive thousands of inputs that all need to be measured within a few milliseconds; those nanosecond delays before an excited fluorophore emits light add up. "Nanoseconds don't sound like a lot of time, but a 1 megapixel image - that's sort of 'potato quality' on YouTube these dayswould be 1000x1000 pixels: that's a million measurements," he says.

To increase imaging speed, labs are working to parallelize the imaging process. For example, rather than serially scanning every single pixel in the image, it's possible to build random access microscopes that only fire at areas of interest, such as neurons, thus reducing the pixels that need to be scanned from a million to a few thousand. More recently, Podgorski has been taking an approach well known outside of biology in fields such as communications and astronomy: compression. Images and videos have structure-details such as edges, or areas with the same color. "If you know the structure of a space of images, you can compress it," he says. There are limits to how a brain can possibly look under a microscope, which makes it possible to model what activity can be expected from a particular area; the microscope need only measure say, the active neurons present.

With his faster compressive $2 \mathrm{P}$ microscope, Podgorski has been able to measure glutamate inputs-the major excitatory neurotransmitter in mammalsacross sections of dendritic arbors, finding correlations in patterns of activity, at least in anesthetized animals, that are spatially compartmentalized to different parts of the dendrite ${ }^{2}$.

There are also efforts to capture more of the brain at a time, moving from small patches of a few hundred microns on a single imaging plane to millimeter areas across volumes of tissue. A hybrid multiphoton microscope built by Alipasha Vaziri's's lab at Rockefeller University, for example, has managed to image 12,000 mouse neurons at single-cell resolution through an entire cortical column ${ }^{3}$. Iterating through multiple planes is not trivial, says Waters, but there are emerging optical means to shift the laser's focus quickly, as well as approaches such as temporal multiplexing, which splits a laser pulse into

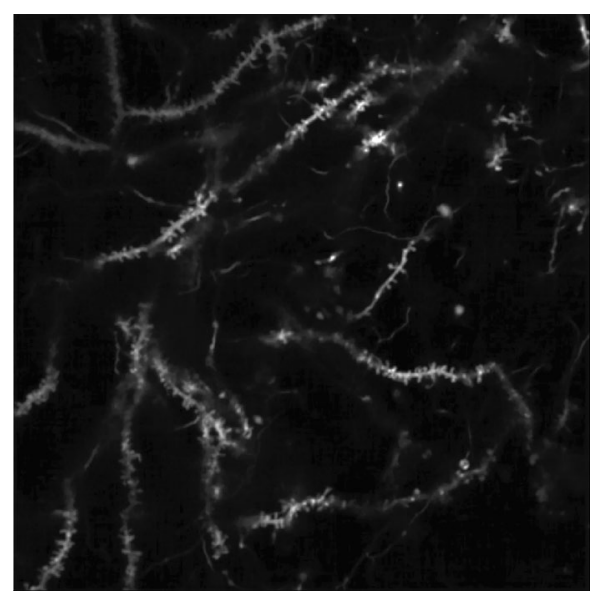

The need for speed | High-speed 2P microscopy enables imaging of the dendritic spines of mouse neurons. Credit: K. Podgorski 
multiple streams that can target different depths. Waters' lab is currently working on applying such approaches to 3P systems, which at the moment are just a little slower than their $2 \mathrm{P}$ predecessors.

"Where 2 photon goes, 3 photon will most certainly follow," says Waters.

\section{All in the animal}

For all the technical inputs and optimizations required for multiphoton microscopy, much attention must be paid to the animals themselves. "The actual imaging is probably not the most challenging thing," says Pucak. "The challenging thing is getting good at the surgery, and getting the appropriate animal in the first place." Animals first need to be made that express the desired fluorescent molecules at the structures of interest; for a mouse, where the necessary transgenic tools are best established, that can take months.

Then there's that thick, imaging-unfriendly bone to content with. 3P instruments, which their longer, less scattering wavelengths, may eventually eliminate or reduce the need for surgeries to remove bone ${ }^{4}$, (and in larger animals such as rat, the dura underneath) and implant cranial windows and coverslips, but for now, those procedures must go on. That surgery takes practice, as it's important to minimize things like gliosis and scarring, which can obscure details and limit depth and resolution, says Pucak. Beyond the brain, some labs are using $2 \mathrm{P}$ microscopes to image neurons in the spine; while studying multiple sclerosis (MS) mouse models, for example, the myelinated white matter of interest is located on the outside of the spinal cord, rather than deep within the brain, which makes it more accessible to $2 \mathrm{P}$ microscopes. The surgical preparation to remove parts of the spinal column, however, can be more challenging to perform than installing a cranial window on the skull.

Then add some time for acclimation and training. If the animal is to be awake during imaging, which lets researchers measure neuronal responses to stimuli, such as whisker wiggles or visual or olfactory inputs, it will then need some time to get used to the head stage; others may be trained to perform a task under the microscope.

With fluorescent transgenes expressed, windows installed, and animals ready, imaging under the microscope can begin. There, the subject needs to be made comfortable; animals, whether anesthetized, head- (or spine-) fixed, or freely moving, all need support and monitoring to minimize stress.

Compared to cell cultures and ex vivo preparations, living animals offer researchers the opportunity to study biological phenomena in its full biological context. But animals have the pesky habit of moving. "Even if the animal is anesthetized, it's breathing," says Pucak. If you're trying to visualize tiny structures, such as dendritic spines, even a tiny bit of motion can be problematic. Here, post-hoc corrections with software can correct for motion, though corrections can be more complicated with awake and freely moving animals.

Motion is nevertheless a variable that researchers are increasingly hoping to capture. "The central point of the central nervous system is to information the behavior of the animal-that's its key job," says Damian Wallace, a staff scientist at the Center of Advanced European Studies and Research (Caesar) in Bonn, Germany. Letting the animal behave naturally is informative, but it takes some extra engineering. Microscopes need to be miniaturized and mounted on the animal's head, and the laser pulses must then pass through an optical fiber, which can introduce its own distortions that need to be corrected for. "That's of course just accumulating challenges," says Alexandr Klioutchnikov, a technical assistant at Caesar.

One photon 'miniscopes' with head-mounted cameras have been commercially available and/or relatively easy to construct from open-source guides for a few years now; more complicated $2 \mathrm{P}$ and $3 \mathrm{P}$ versions are now starting to turn up in different labs. For example, Emily Gibson's bioengineering lab at the University of Colorado has built a 2P miniscope for imaging freely behaving mice-such as those following a scent plume, to record how olfactory neurons respond. The initial miniscope weighed just 2.5 grams $^{5}$; recent modifications have brought that to 1.8 grams, she says. That is a bearable load for a mouse, and, in a future collaboration with Zoe Donaldson's lab at UC Boulder, prairie voles, which don't take well to head fixation and have unique social behaviors that necessitate free movement.

Last summer, Gibson, her collaborator Diego Restrepo, and Intelligent Imaging Innovations Inc received a Small Business Innovation Grant to commercial the 2P device. Imaging depth, however, tends to be a little lower in awake, moving animals-reduced down to about 200-300 micrometers with $2 \mathrm{P}$ miniscopes; $3 \mathrm{P}$ versions to reach greater depths are in the works. Gibson's lab is working on such a device for mice, she says, while Wallace and Klioutchnikov at Caesar focused their initial 3P engineering efforts on a larger rodent capable of bearing a little more

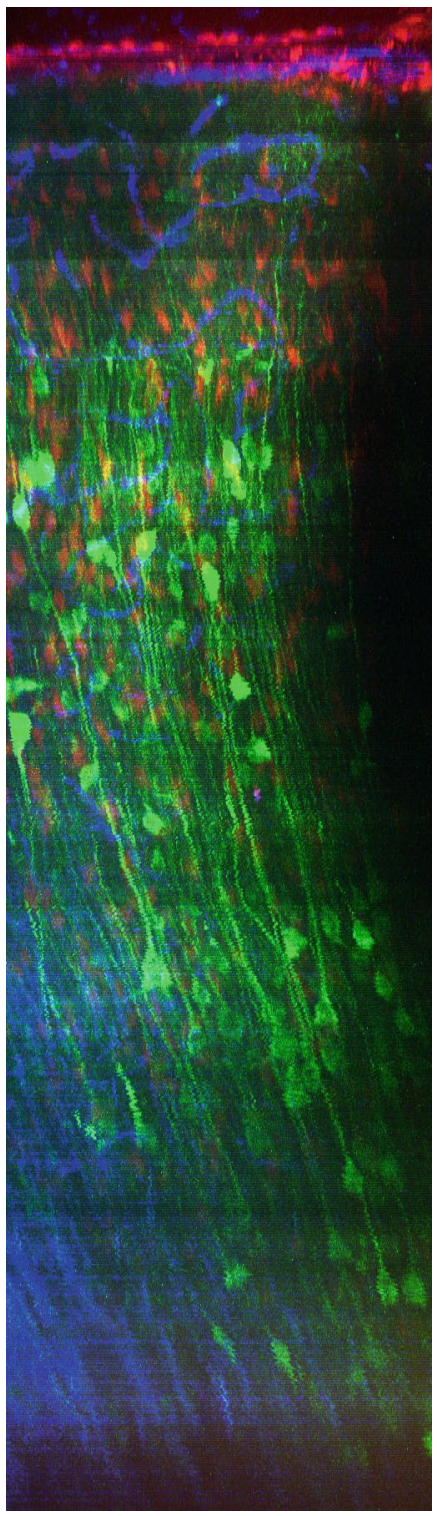

All together | A compiled volume of recordings from the rat cortex, taken with a $3 \mathrm{P}$ microscope. Credit: A. Klioutchnikov

weight: the rat $^{6}$. Mice are tempting says Klioutchnikov; the 3P miniscope will just need further miniaturizing to make it suitable for a mouse.

\section{Power on}

3P microscopy isn't just limited to mice. "People think of 3 photon as being particularly useful for imaging deep into thick [mouse] specimens, and then they're thinking larger animals... being able to image through the full thickness of cortex in rats, and even going into macaques," says Podgorski. "But 3 photon has also been super useful in fruit flies." In flies, for example, the $3 \mathrm{P}$ lasers are able to make it through the insect's thick cuticle ${ }^{7,8}$. 


\section{Box 1 | Building better indicators}

An image will only be as good as its indicator. While the classic GFP suffices in many applications, many are pushing the limits of fluorescent indicators to go along with advances in microscope technologies. With collaborators at Janelia and beyond, Podgorski has been working to improve existing indicators and build better ones: faster calcium indicators, a new generation of glutamate indicators, and new modalities that can sense other neurotransmitters, such as sensors for GABA and acetylcholine. "We want to have in the tool box ways to measure all these different channels of communication," he says.

To build an indicator, you need to measure lots of variants, as something that performs well in vitro doesn't always stand up in in vivo. There, you have to optimize affinities for the sensor and make sure the ranges used reflect the actual concentrations of substrates found in an animal. The indicator needs to be bright enough to seen and fast enough to measure the activity in question, and it has to make it to the right part of a cell and operate at physiologically relevant temperatures.

Voltage dye indicators are also in the works; these molecules can better capture the millisecond \& sub-millisecond time scales that neurons fire at, but imaging these, particularly across wide fields of view and different brain regions, will take faster microscopes. "We're not going to get there with scanning," says Waters. "We need to come up with much more clever ways to generate images."
Murat Yildirim, a postdoctoral fellow working with Peter So and Mriganka Sur at MIT who recently built a 3P microscope that could record evoked neural activity in all 6 layers of the mouse visual cortex and into the white matter beneath ${ }^{9}$, is looking to apply the technology not just in mice, but also in smaller animals in the future: flies, worms, even Hydra, as the genetic toolboxes for these animals expand. A 3P microscope may still only reach a short distance into the cortex of a large macaque, but it could capture larger volumes of the nervous system in smaller animals. In zebrafish for example, a 3P system made it through the opaque skulls of adult fish to image the entire forebrain and deep into the cerebellum, reaching depths of $750 \mu \mathrm{m}^{10}$.

But with great power comes great responsibility: to compensate for the lack of efficiency with added photons, researchers need to push greater power into a tighter time space-from microseconds to femtoseconds, says Podgorski; that runs the risk of photodamaging tissue. "As neuroscientists and as other scientists are pushing the boundaries on how much they want to measure in a given subject, we're using higher and higher laser powers, we're putting more energy into the brain, and at some point you start to do damage and you need to know when that's occurring," he says.

Those thresholds can vary between organisms: while a mouse brain might handle 250 milliwatts of power, a more sensitive fly might only tolerate 15 to 20 milliwatts. "You have to know in advance how deep you want to image, and then you pick the technology to suit the experiment," he says.

As researchers continue to push the limits of 2P \& 3P microscopes-their depth, speed, and scale, plus the development of new and better indicators (Box 1), some questions remain about accessibility. "It's very hard to share complicated instruments," says Podgorski. "It's so satisfying when we make a new fluorescent indicator and someone wants to use it and we just put it in the mail. We can't do that with microscopes."

There are moves in two directions in the field: to make instruments cheaper, and to set up observatories. The Advanced Imaging Center at Janelia funds researchers to in and use its instruments, for example. Lasers, particularly $3 \mathrm{P}$ versions, aren't cheap; the commercial microscopes to go with them can sometimes lack flexibility, while specialized scopes can be tricky for those without engineering backgrounds to construct-Yildirim likens it to a restaurant chef, who must consistently serve a wide range of tastes vs. the family cook who can tweak and experiment at home.

"In engineering you can develop many, many microscopes," he says. But an important question remains: how can you transfer those to neuroscience labs? Engineers need to demonstrate that their microscopes are more than just proof of the latest concept, but that they can stand up to repeated, long-term use that scientists need to perform their experiments, he says.

\section{The field is set?}

The scientific applications that multiphoton microscopes facilitate are nevertheless forging on. "We're in that phase where we understand that we've made some big advances as a field, and those first experiments to answer biological questions are ongoing," say Waters. "As a cortical physiologist, just being able to see into the deep layers of cortex has been pretty exciting."

With their 3P miniscope, Wallace and Klioutchnikov could image through the cortical mantel of their rats, which expands the horizons of questions that can be answered in a freely behaving animal. Neurons that project out to influence behavior tend to be deeper neurons, Wallace says, "To be able to get access to those with imaging technologies is a huge advantage."

At Johns Hopkins, Pucak is excited by the different applications her users bring to the Core's different options. Those same $2 \mathrm{P}$ lasers can also, for example, be used for intentional damage, such as killing a single glial cell; that animal can then be brought back repeatedly to follow how other cells in the brain respond to that damage. "You can sit and watch cells move," she says. "You can watch cells divide in real time in an actual brain."

Whether imaging shallow GFPexpressing cells under a confocal or pushing depth limits with $3 \mathrm{P}$ microscopes, the images she sees never get old. "I never ever get tired of it," Pucak says. "You can do it every single day, and you're still like 'wow, look at this'. It's still exciting to see."

Ellen P. Neff $₫$
Lab Animal.
$\bigotimes_{\text {e-mail: ellen.neff@us.nature.com }}$

Published online: 12 August 2020

https://doi.org/10.1038/s41684-020-0623-0

References

1. Takasaki, K., Abbasi-Asl, R, and Waters, J. eNeuro 7, ENEURO.0255-19.2019 (2020)

2. Kazemipour, A. et al. Nat. Method. 16, 778-786 (2019)

3. Weisenburger, S. et al. Cell 177, 1050-1066.e14 (2019).

4. Wang, T. et al. Nat. Method. 15, 789-792 (2018).

5. Ozbay, B. N. et al. Sci. Rep. 8, 8108 (2018).

6. Klioutchnikov, A. et al. Nat. Method. 17, 509-513 (2020).

7. Tao, X. et al. Biomed. Opt. Express 8, 1277-1289 (2017).

8. Aragon, M.J. et al. bioRxiv (2020). https://doi.org/10.1101/798686

9. Yildirim, M., Sugihara, H., So, P. T. C. \& Sur, M. Nat. Commun. 10, 177 (2019).

10. Chow, D. M. et al. Nat. Method. 17, 605-608 (2020). 